Supporting Information

\title{
Modulating Stereoselectivity through Electrostatic Interactions in a SPINOL-Phosphoric Acid Catalyzed Synthesis of 2,3-Dihydroquinazolinones
}

\author{
Croix J. Laconsay, ${ }^{\ddagger}$ Trevor J. Seguin, ${ }^{\ddagger}$ and Steven E. Wheeler ${ }^{\ddagger \S^{*}}$ \\ ${ }^{\ddagger}$ Department of Chemistry, Texas A\&M University, College Station, TX 77842 \\ $\S$ Department of Chemistry, University of Georgia, Athens, GA 30602 \\ "E-mail: swheele2@uga.edu
}




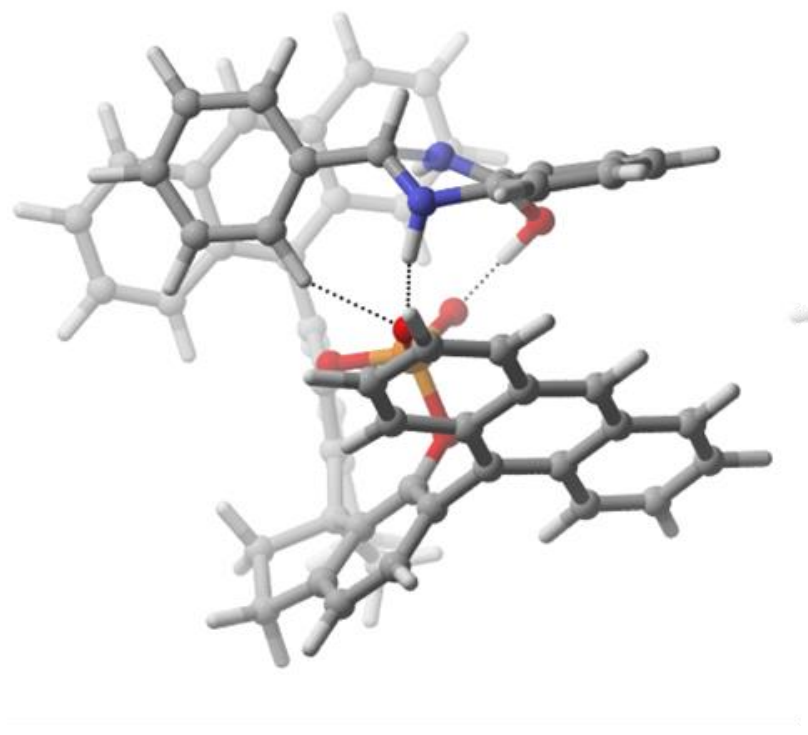

8.4

Activation Mode 1

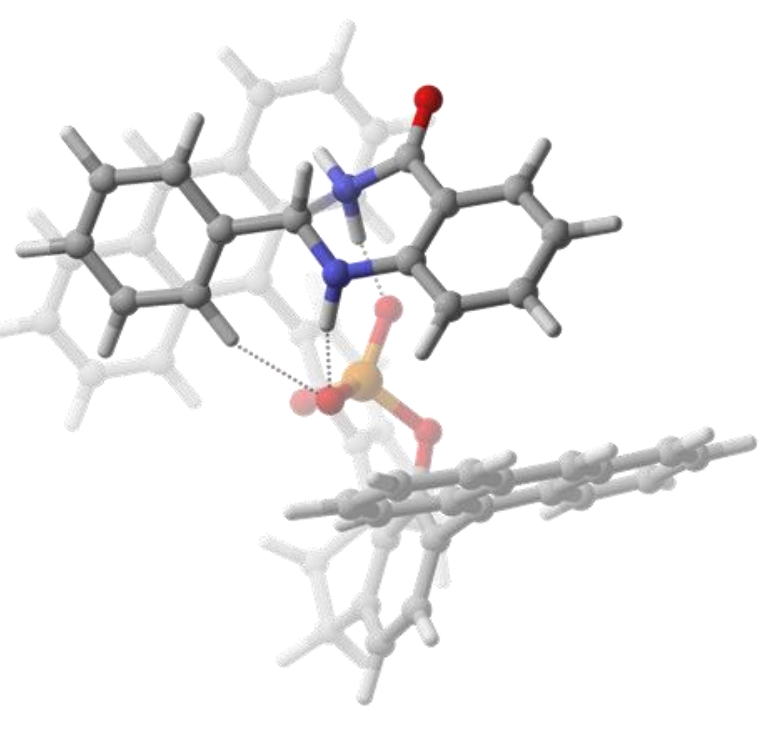

TS(E)

Activation Mode 2

Figure S1. Optimized TS structures of activation modes 1 and 2 for Entry 6 in Table 1 of the main text, along with relative free energies in $\mathrm{kcal} / \mathrm{mol}$. 
Table S1. Absolute and relative free energies for all computed TS structures. The lowest-lying (S) and (R)-structures for each system are in bold.

\begin{tabular}{|c|c|c|c|c|c|c|}
\hline $\mathrm{R}$ & PA & TS Name & G (Hartrees) & $\Delta \Delta \mathrm{G}^{\ddagger}(\mathrm{kcal} / \mathrm{mol})$ & $\begin{array}{c}\text { Stereochemistry } \\
\text { of product }\end{array}$ & $\begin{array}{l}\text { Configuration } \\
\text { of imminium }\end{array}$ \\
\hline \multirow[t]{4}{*}{$p-\mathrm{BrPh}$} & 1 & TS1 & -5217.675014 & 0.0 & $\mathbf{S}$ & $\mathbf{E}$ \\
\hline & & TS2 & -5217.674787 & 0.1 & $\mathbf{R}$ & $\mathbf{Z}$ \\
\hline & & TS3 & -5217.674318 & 0.4 & $\mathrm{~S}$ & $\mathrm{Z}$ \\
\hline & & TS4 & -5217.672891 & 1.3 & $\mathrm{R}$ & $\mathrm{E}$ \\
\hline \multirow[t]{4}{*}{$p-\mathrm{BrPh}$} & 2 & TS5 & -5060.546465 & 0.0 & $\mathbf{S}$ & $\mathbf{E}$ \\
\hline & & TS6 & -5060.543589 & 1.8 & $\mathbf{R}$ & $\mathbf{Z}$ \\
\hline & & TS7 & -5060.543400 & 1.9 & $\mathrm{~S}$ & $\mathrm{Z}$ \\
\hline & & TS8 & -5060.540580 & 3.7 & $\mathrm{R}$ & $\mathrm{E}$ \\
\hline \multirow[t]{8}{*}{$m-\mathrm{BrPh}$} & 3 & TS9 & -5674.742455 & 0.0 & $\mathbf{S}$ & $\mathbf{E}$ \\
\hline & & TS10 & -5674.742364 & 0.1 & $\mathrm{~S}$ & $\mathrm{E}$ \\
\hline & & TS11 & -5674.739343 & 2.0 & $\mathrm{~S}$ & $\mathrm{Z}$ \\
\hline & & TS12 & -5674.739248 & 2.0 & $\mathrm{~S}$ & $\mathrm{Z}$ \\
\hline & & TS13 & -5674.738137 & 2.7 & $\mathbf{R}$ & $\mathbf{Z}$ \\
\hline & & TS14 & -5674.736796 & 3.6 & $\mathrm{R}$ & $\mathrm{E}$ \\
\hline & & TS15 & -5674.736400 & 3.8 & $\mathrm{R}$ & $\mathrm{Z}$ \\
\hline & & TS16 & -5674.735784 & 4.2 & $\mathrm{R}$ & $\mathrm{E}$ \\
\hline \multirow[t]{4}{*}{$p-\mathrm{BrPh}$} & 3 & TS17 & -5674.741934 & 0.0 & $\mathbf{S}$ & $\mathbf{E}$ \\
\hline & & TS18 & -5674.739252 & 1.7 & $\mathrm{~S}$ & $\mathrm{Z}$ \\
\hline & & TS19 & -5674.737075 & 3.0 & $\mathbf{R}$ & $\mathbf{E}$ \\
\hline & & TS20 & -5674.736683 & 3.3 & $\mathrm{R}$ & $\mathrm{Z}$ \\
\hline \multirow[t]{8}{*}{$p-\mathrm{OMePh}$} & 3 & TS21 & -3214.333793 & 0.0 & $\mathbf{S}$ & $\mathbf{E}$ \\
\hline & & TS22 & -3214.333611 & 0.1 & $\mathrm{~S}$ & $\mathrm{E}$ \\
\hline & & TS23 & -3214.331230 & 1.6 & $\mathrm{~S}$ & $\mathrm{Z}$ \\
\hline & & TS24 & -3214.330533 & 2.0 & $S$ & $\mathrm{Z}$ \\
\hline & & TS25 & -3214.329291 & 2.8 & $\mathbf{R}$ & $\mathbf{E}$ \\
\hline & & TS26 & -3214.329022 & 3.0 & $\mathrm{R}$ & $\mathrm{Z}$ \\
\hline & & TS27 & -3214.329013 & 3.0 & $\mathrm{R}$ & $\mathrm{Z}$ \\
\hline & & TS28 & -3214.328235 & 3.5 & $\mathrm{R}$ & $\mathrm{E}$ \\
\hline \multirow[t]{4}{*}{$\mathrm{Ph}$} & 3 & TS29 & -3099.854224 & 0.0 & $\mathbf{S}$ & $\mathbf{E}$ \\
\hline & & TS30 & -3099.852716 & 0.9 & $\mathrm{~S}$ & $\mathrm{Z}$ \\
\hline & & TS31 & -3099.850799 & 2.1 & $\mathbf{R}$ & $\mathbf{Z}$ \\
\hline & & TS32 & -3099.849174 & 3.2 & $\mathrm{R}$ & $\mathrm{E}$ \\
\hline
\end{tabular}




\begin{tabular}{|c|c|c|c|c|c|}
\hline \multirow{17}{*}{\multicolumn{2}{|c|}{$m$-OMePh }} & TS33 & -3214.335491 & 0.0 & $\mathbf{S}$ \\
\hline & & TS34 & -3214.331312 & 2.6 & $\mathrm{~S}$ \\
\hline & & TS35 & -3214.330932 & 2.9 & $S$ \\
\hline & & TS36 & -3214.330522 & 3.1 & $S$ \\
\hline & & TS37 & -3214.330492 & 3.1 & $\mathrm{~S}$ \\
\hline & & TS38 & -3214.330320 & 3.2 & $\mathbf{R}$ \\
\hline & & TS39 & -3214.329527 & 3.7 & $\mathrm{~S}$ \\
\hline & & TS40 & -3214.329508 & 3.8 & $\mathrm{~S}$ \\
\hline & & TS41 & -3214.328965 & 4.1 & $\mathrm{~S}$ \\
\hline & & TS42 & -3214.328786 & 4.2 & $\mathrm{R}$ \\
\hline & & TS43 & -3214.328498 & 4.4 & $\mathrm{R}$ \\
\hline & & TS44 & -3214.327998 & 4.7 & $\mathrm{R}$ \\
\hline & & TS45 & -3214.326959 & 5.4 & $\mathrm{R}$ \\
\hline & & TS46 & -3214.326670 & 5.5 & $\mathrm{R}$ \\
\hline & & TS47 & -3214.325652 & 6.2 & $\mathrm{R}$ \\
\hline & & TS48 & -3214.325084 & 6.5 & $\mathrm{R}$ \\
\hline & & TS49 & -3214.324731 & 6.8 & $\mathrm{R}$ \\
\hline \multirow[t]{13}{*}{$o-\mathrm{OMePh}$} & \multirow[t]{13}{*}{3} & TS50 & -3214.333150 & 0.0 & $\mathbf{S}$ \\
\hline & & TS51 & -3214.332789 & 0.2 & $S$ \\
\hline & & TS52 & -3214.327315 & 3.7 & $\mathrm{~S}$ \\
\hline & & TS53 & -3214.326636 & 4.1 & $\mathbf{R}$ \\
\hline & & TS54 & -3214.326633 & 4.1 & $\mathrm{R}$ \\
\hline & & TS55 & -3214.325558 & 4.8 & $\mathrm{R}$ \\
\hline & & TS56 & -3214.325396 & 4.9 & $\mathrm{R}$ \\
\hline & & TS57 & -3214.325054 & 5.1 & $\mathrm{~S}$ \\
\hline & & TS58 & -3214.323359 & 6.1 & $\mathrm{R}$ \\
\hline & & TS59 & -3214.323058 & 6.3 & $\mathrm{R}$ \\
\hline & & TS60 & -3214.320061 & 8.2 & $S$ \\
\hline & & TS61 & -3214.319978 & 8.3 & $\mathrm{R}$ \\
\hline & & TS62 & -3214.319178 & 8.8 & $\mathrm{R}$ \\
\hline \multirow[t]{7}{*}{$o-\mathrm{NO}_{2} \mathrm{Ph}$} & \multirow[t]{7}{*}{3} & TS63 & -3304.337072 & 0.0 & $\mathbf{S}$ \\
\hline & & TS64 & -3304.336021 & 0.7 & $\mathrm{~S}$ \\
\hline & & TS65 & -3304.332806 & 2.7 & $\mathrm{~S}$ \\
\hline & & TS66 & -3304.330827 & 3.9 & $\mathbf{R}$ \\
\hline & & TS67 & -3304.327769 & 5.8 & $\mathrm{R}$ \\
\hline & & TS68 & -3304.327396 & 6.1 & $\mathrm{R}$ \\
\hline & & TS69 & -3304.327030 & 6.3 & $\mathrm{R}$ \\
\hline
\end{tabular}




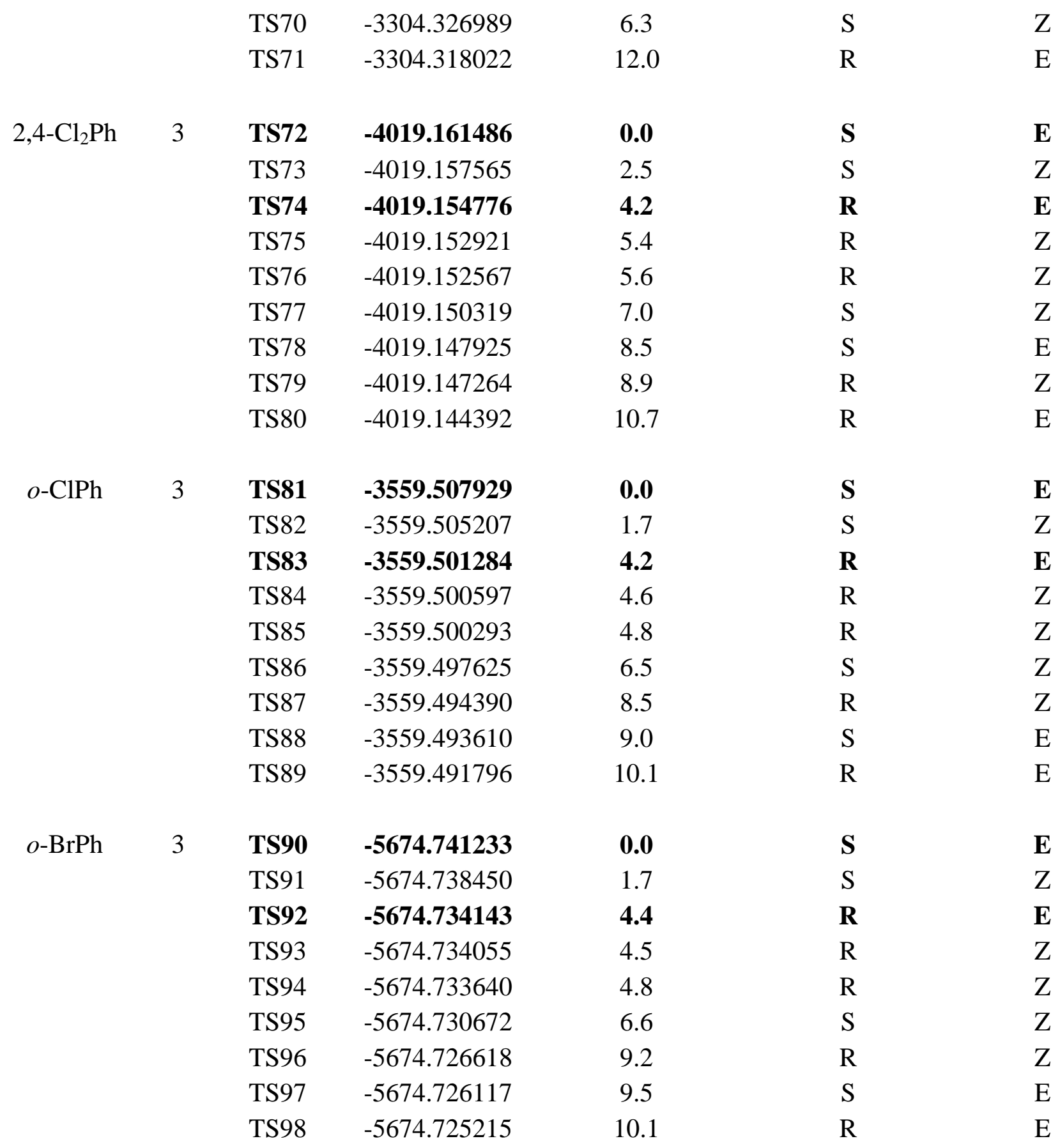

See SI_Cartesian_Coordinates.xyz for optimized coordinates of each TS structure listed above. 